6. Frazer, W. R. Phys. Rev. 115, p. 1763 (1959).

7. Drell, S. D. (Session S 1 of this Conference.)

8. Cabibbo, N. and Gatto, R. Phys. Rev. Letters 4, p. 313 (1960).

9. Samios, N. (Session S 1 of this Conference ; discussed also by Ashkin in Session R-1.)

10. Wong, H. S. Phys. Rev. Letters 5, p. 70 (1960).

11. Berman, S. M. and Geffen, D. A. Preprint from Institute for Theoretical Physics, Copenhagen, Denmark.

12. Ball, J. Phys. Rev. Letters 5, p. 73 (1960).

13. Gourdin, Lurié, and Martin, CERN preprint (1960).

14. Chew, Goldberger, Low and Nambu. Phys. Rev. 106, p. 1345 (1957).

15. Harris, Orear and Taylor. Phys. Rev. 106, p. 327 (1957).

\title{
THE ANOMALOUS MAGNETIC MOMENT OF THE MUON
}

\author{
G. Charpak, F. J. M. Farley, R. L. Garwin ${ }^{(*)}$, T. Muller, J. C. Sens, V. L. Telegdi ${ }^{(* *)}$, C. M. York ${ }^{(* * *)}$ \\ and A. Zichichi
}

CERN, Geneva, Switzerland

(presented by C. M. York)

\section{INTRODUCTION}

The puzzle of the muon is known to all: how can a charged particle be 207 times as heavy as the electron, but be identical with the electron in all other respects (absence of strong interactions, same weak interaction coupling constants, etc.)? The mystery deepened in January 1957 when it was shown ${ }^{1)}$ in the demonstration of non-conservation of parity by precession of muons at rest that the magnetic moment of the muon was within $5 \%$ that appropriate to a Dirac particle of the muon mass-i.e., one muon magneton $\mu_{0}=\frac{e \hbar}{2 m c}$. If one then grants that the muon is to be described, as well as the electron, by the Dirac equation, then the magnetic moment is of course to be approximately 1 (the " $g$-factor" $\left.\mu /\left(s \mu_{0}\right)=2\right)$ and it becomes of great interest to see whether the muon $g$-factor is exactly as predicted by quantum electrodynamics or not $(g / 2=1+\alpha / 2 \pi+\ldots)$ since the muon magnetic moment anomaly $(g-2) / 2$ arises from interaction with photons of energy comparable to the muon rest mass (i.e. $100 \mathrm{MeV}$ ) rather than $0.5 \mathrm{MeV}$ in the case of the electron; involving distances comparable to the muon or electron Compton wavelength respectively-i.e. $2 \times 10^{-13} \mathrm{~cm}$ for the muon. Thus one has a sensitivity to deviations from the Coulomb law in this order of magnitude of distance (already well established by Hofstadter et al to smaller distances) and to the muon form factor with respect to the electromagnetic field. The latter can be represented by an extension in space or a cut-off in the coupling to the e.m. field at a photon energy $\Lambda m c^{2}$ which has been shown ${ }^{2)}$ to give rise to a change in the muon moment anomaly of the form

$$
(g-2) / 2 \equiv a=\frac{\alpha}{2 \pi}\left[1-\frac{2}{3} \Lambda^{-2}\right]+0.75 \frac{\alpha^{2}}{\pi^{2}}
$$

(*) Ford Foundation Fellow, from IBM Watson Laboratory, Columbia University, New York.

(**) NSF Senior Fellow, on leave from University of Chicago.

$(* * *)$ Ford Foundation Fellow, presently Dept. of Physics, UCLA, Los Angeles, California. 
Thus a $g$-factor experiment to an accuracy worse than $0.1 \%$ does not test the muon-photon interaction at all (although it does give some information on other interactions of the muon), while an experiment giving $g$ to an accuracy of $10^{-5}(1 \%$ of $\alpha / 2 \pi)$ is sensitive to anomalies in the coupling at energies as high as $1,000 \mathrm{MeV}$. Even a result in complete agreement with the QED prediction would be of considerable interest because of the restrictions it would put on attempts to explain the muon-electron mass difference.

It is of interest to note that the $\alpha^{2}$ term for the QED anomaly of the muon differs from that of the electron because of the existence of electron pairs ${ }^{3)}$ in the correction of this order to the muon moment. But this diagram has accidentally a small coefficient, and the $\alpha^{2}$ term would not show up in a $1 \%$ of $\alpha / 2 \pi$ experiment. Thus it is of very considerable interest to determine the muon $g$-factor to one part in $10^{5}$.

\section{KNOWLEDGE OF $g-2$ BY INDIRECT METHODS}

It is of course possible to refine the first measurements of precession rate by going to higher fields, direct comparison with the proton nuclear resonance frequency in the same field, etc. But such improvements still give only the muon moment, and not the $g$-factor (which is twice the moment in muon magnetons). But to obtain the muon moment anomaly from measurements of the moment it is necessary to know the mass well enough to compute the muon magneton to the desired accuracy. It is possible to measure the mass relativity accurately by making use of the natural width of the $K$-edge of lead, which exactly coincides with the photon emitted from the $3 D-2 P$ transition of $\mu^{-}$in phosphorus. Koslov, Fitch, and Rainwater ${ }^{4)}$ first used this pair of target and absorber to set a very useful lower limit on the muon mass, and the group at Chicago have just published ${ }^{5)}$ an actual measurement making use of the breadth of the $K$-edge. The result is $m_{\mu} / m_{e}=$ $=206.76_{-0.03}^{+0.02}$. Probably there are no large systematic errors in this measurement, and the calculation of the atomic energy levels should certainly be good to this accuracy. A similar experiment has later been done at Columbia, but the answer is not known to me.
The latest value of the magnetic moment, as yet unpublished, has been obtained by Hutchinson, Menes, Patlach, Penman and Shapiro at Columbia in an experiment which is an improvement of that published ${ }^{6)}$ previously.

This is a beat-frequency precession measurement of the moment, differing from the previously published result in being done at much higher field and with completely different apparatus. Table I compares the two experiments

Table I. Comparison of $\mu$ magnetic momert measurements

\begin{tabular}{|c|c|c|}
\hline & New & $\begin{array}{l}\text { Phys. Rev. Letters } \\
\text { 2, p. } 516 \text { (1959) }\end{array}$ \\
\hline Frequency . . . & $178 \mathrm{Mc}$ & $86 \mathrm{Mc}$ \\
\hline Magnet stability . & $\begin{array}{l}10^{-7} \text { (locked to } \\
\text { proton reso- } \\
\text { nance line) }\end{array}$ & $3 \times 10^{-5}$ \\
\hline Homogeneity . & $2 \times 10^{-5}$ & $10^{-4}$ \\
\hline Timing . . . . & 1 ns full-width & $1 \mathrm{~ns}$ \\
\hline Target . . . . & $\mathrm{CH}_{2} \mathrm{I}_{2}$ & $\mathrm{CHBr}_{3}$ \\
\hline Fast counters . . & separate $\mu$ and $e$ & same $\mu$ and $e$ \\
\hline Time measurement & pulse train & rung oscillators \\
\hline Result $f_{\mu} / f_{p}$ & $3.1832 \pm 0.0002$ & $3.1834 \pm 0.0002$ \\
\hline
\end{tabular}

The two results are entirely consistent and should thus be averaged to give $f_{\mu} / f_{p}=3.1833 \pm 0.00013$. More running time can reduce this error at least by a factor two, but it is unlikely that the mass measurement can ever be improved to comparable accuracy. Thus at present $(g-2) / 2=\alpha / 2 \pi\left(2.0_{-0.10}^{+0.14}\right)$, by a quite indirect but hopefully reliable method.

\section{DIRECT $g-2$ EXPERIMENT}

Immediately upon the determination that $g \simeq 2$, it was recognized that one could take advantage of the similarity of spin and orbit motion for particles with $g$-2 in static magnetic fields in order to measure the deviation of $g$ from 2, without requiring any accurate knowledge of the mass. Such an experiment has been done for the free electron by Shupp, Pidd and Crane ${ }^{7)}$ with results of accuracy $2 \times 10^{-3}$ of $\alpha / 2 \pi$.

The principle of all such experiments is shown in Fig. 1. 


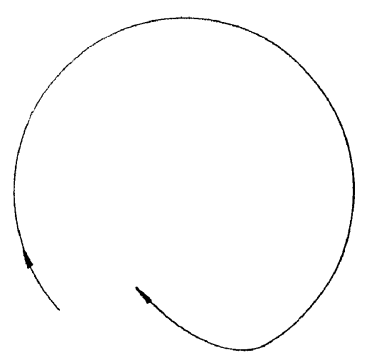

(a) $g=2$

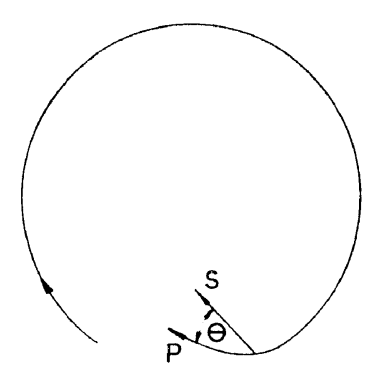

(b) $g=2(1+a)$
Fig. 1 The principle of measuring $(g-2)$ by precession in a static magnetic field.

The angle $\theta$ by which an initially longitudinal spin precesses away from the momentum for circular orbits may be calculated as
I. $\theta=2 \pi N a \gamma \quad$ or
II. $\theta=\omega_{0}$ Bat

where $N$ is the number of turns the particle makes in the field, $B$ the magnitude of the field, $a=(g-2) / 2$, $t$ the time, $\omega_{0}$ is the cyclotron frequency of low energy mesons in a 1 gauss field (i.e. $13.5 \mathrm{kc}$ ), and $\gamma$ the ratio of the total energy to the rest mass. To detect $g-2$ is not hard. To measure it one has an experiment of the type I or II, according as to whether it is easier to store muons for a known number of turns (some long magnetic channel) or for a known time interval. At CERN we have now a great deal of experience with methods of the type II and even some observations of $g-2$ in a magnet with about 100 turns. On the other hand, we have also built a type I appa-

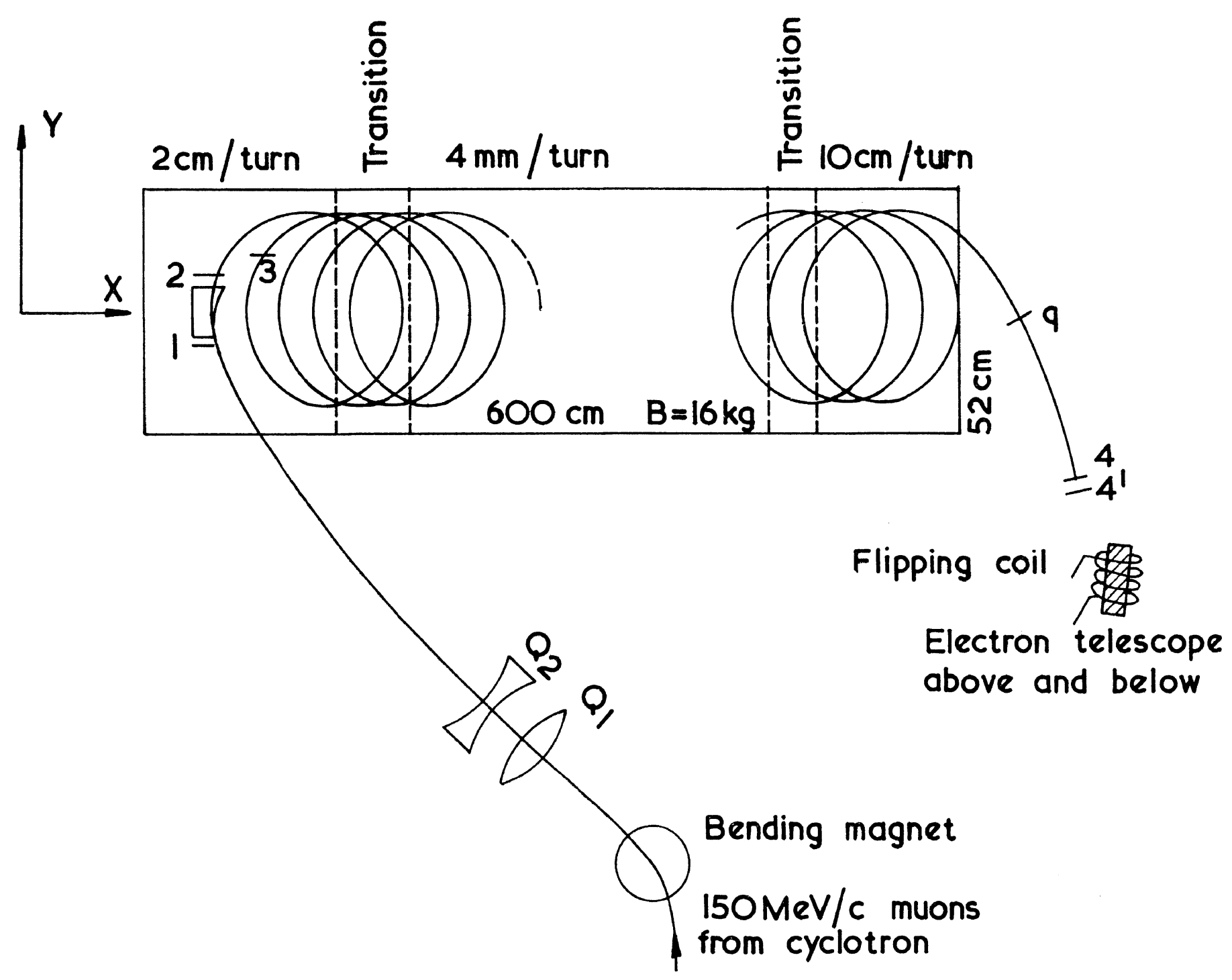

Fig. 2 Diagram of the proposed experiment using the 6 meter magnet. 
ratus of 20 turns (a focussing helix invented by M. G. N. Hine and developed by Lederman, Panofsky and Telegdi), but we have not yet used it.

For the last year our goal has been a measurement of $g-2$ to a precision of $1 \%$ of $\alpha / 2 \pi$, and I have only time to describe this particular technique and some preliminary results obtained with it. There is a considerable body of analysis available of this experiment, but it is not possible here to give more than a description. There are very many possible methods for storing muons for a long time in magnetic fields. They differ in size, cost, systematic errors, and in the techniques on which they rely. Our method seems quite reasonable to us. We do not claim that that it is superior to any other.

If one uses an iron-core magnet one is limited to about 16,000 gauss - a muon precession frequency of $200 \mathrm{mc}$. Thus one has $\theta=2 \pi$ in 4.5 microseconds. It is reasonable to store the muons for 6-7 microseconds (almost three mean lives for our muons) so that the precession due to $g-2$ is about $500^{\circ}$. Thus a $1 \%$ experiment involves measuring the spin with respect to momentum to an accuracy of $5^{\circ}$. In $6 \mu \mathrm{sec}$ the muons go about $1 \mathrm{~km}$. One has available at most $10^{4}$ muons $/ \mathrm{sec}$ and one has to hit a $(10 \mathrm{~cm})^{2}$ target of the polarization analyzer at a distance of $1 \mathrm{~km}$ (projected). With no vertical or horizontal focussing it would thus require many years of running to count the 3,000 decay electrons required to determine the direction of the spin to the desired accuracy. Things are not so bad and Fig. 2 shows the particular system in use.

The magnet has its field vertical, length $600 \mathrm{~cm}$, width of pole $52 \mathrm{~cm}$. In principle there is a gradient of field in the $y$ direction such that $B_{z}=[1+a y] B_{0}$ which has the consequence that orbits walk to the right with a "step-size" $S=\pi R^{2} a$ per turn. If the field has translational symmetry in $x$, then the orbit " center" remains strictly on the same $y$ as the orbit walks to the right ( $a$ negative). Even for slow changes in gradient there is an adiabatic invariant for the motion -the flux through the orbit, or the central field, and this has the consequence that the orbit center follows always the same contour line of magnetic field. Thus one has to be sure only that the field along the $x$ axis of the magnet is constant and that changes in gradient are reasonably slow to ensure that the orbit does not turn around and walk back along the edge. With our step-size of $4 \mathrm{~mm} /$ turn in the central region, a depression of mean field by $0.1 \%$ results in a $y$-displacement of $2.5 \mathrm{~cm}$, and we have only $\pm 5 \mathrm{~cm}$ available in the storage region.

With increasing storage time there are no particle losses due to motion in the magnet plane (as one makes the magnet longer). Any vertical defocusing would reduce the intensity at the magnet exit exponentially to zero, but it is possible to provide quite strong vertical focusing without interfering with the orbit walking simply by adding a quadratic term to the field

$$
B=B_{0}\left[1+a y+b y^{2}\right]
$$

The linear term provides a very weak "strongfocusing "while the quadratic term gives a relatively strong "weak-focusing" with a vertical oscillation wavelength $\lambda=b^{-\frac{1}{2}}$. Bacause of the periodicity of the focusing forces in time, there are limits to the $a$ and $b$ which may be employed without inducing instabilities in the vertical motion. The lowest stability band is shown in the Fig. 3. The stability band edge sets a limit to the maximum walking speed, i.e., the step size with $b=0$ must be less than $S \leq 0.7 R$ to avoid vertical instability and loss of all particles.

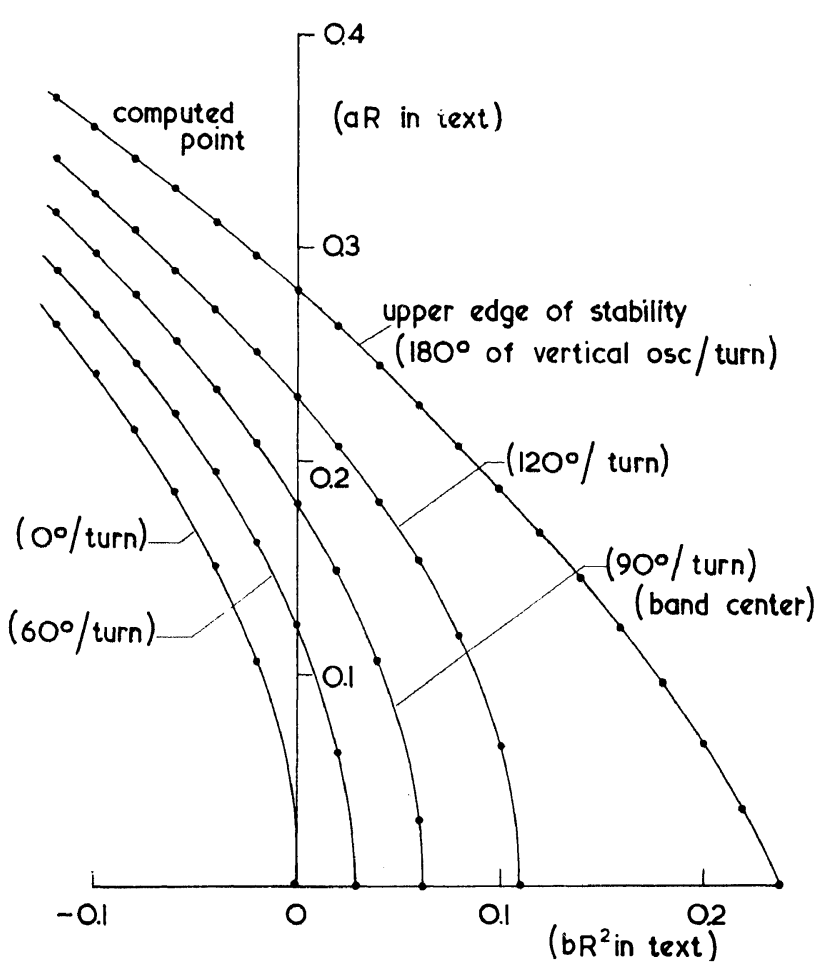

Fig. 3 First stability band for motion in a field illustrated by Eq. (2). The parameters $a$ and $b$ are those appearing in Eq. (2). 
In fact, the $b y^{2}$ term introduces a dependence of the step size $S$ on $y_{0}$, the orbit center, and it turns out that the general step is in the limit of $a, b, c, d$, small

$$
S=\pi R^{2}\left[a+{ }^{3} / 4 c R^{2}+2 b y_{0}+3 d R^{2} y_{0}\right]
$$

with

$$
B=B_{0}\left[1+a y+b y^{2}+c y^{3}+d y^{4}\right]
$$

Alternative formulations for the walking step have also been obtained in terms of integrals over an experimental field distribution. The large dependence of storage time upon $R$ (momentum) can be eliminated by choosing $c=-2 / 3 a R^{-2}$ for low-energy muons, and we have used this value of $c$ to reduce the time spread of storage.

The adiabatic theorem of constant central field will also prevent the orbits from entering or leaving the magnet-a minor difficulty which can be avoided by sharply non-adiabatic changes in field. Thus we make easier non-adiabatic ejection by increasing the step-size (adiabatically) to $10 \mathrm{~cm} /$ turn at the ejection end of the magnet. Then more than half the muons arriving in the ejection region leave the magnet. Since the ejection is by a failure of an adiabatic invariant, the fraction of muons ejected is reduced very much as the ratio of the step-size to the space scale of field change (magnet gap) decreases; computer calculations of orbits show them all crawling around the edge of the magnet for step-size $2 \mathrm{~cm}$ or less.

For injection into the magnet we could indeed use the time-reversed ejection-i.e. if one should focus a beam of $20 \mathrm{MeV}$ muons into the ejection region one will find a considerable fraction of them stored in the magnet. $20 \mathrm{MeV}$ muons are scarcer than those of $80 \mathrm{MeV}$, and we find it more convenient to moderate in the magnet muons of $80 \mathrm{MeV}$. Moderation has the disadvantage of increasing the $\Delta p / p$ of a beam by $\left(p_{0} / p\right)^{7 / 4}$ because of the steep increase of ionization loss at small energy, and it involves also multiple scattering ( 0.1 radians) in beryllium, but we have experience with it and it works. Thus in Fig. 2 the incident longitudinally polarized muon beam from the cyclotron is focused by the quadrupoles onto the moderator. A band of muons of momentum $87 \mathrm{MeV} / \mathrm{c} \pm 10 \%$ then has a step-size of $2 \mathrm{~cm}$ so that the particles clear the moderator and walk toward the storage region. About $1 / 3$ of the particles are within the vertical acceptance aperture, and the spread in the center position is $\pm 2 \mathrm{~cm}$. As the orbits drift down the magnet the effective gradient is reduced by a factor of 5 (and the synchronizing term added) so that Liouville's theorem predicts an expansion of the bundle centers to $\pm 10 \mathrm{~cm}$. Thus one loses an additional factor of 2 . No further losses except a factor of 10 by decay occur before the muons reach the end of the magnet. Thus about $1 \%$ of the injected muons focussed on the $(2 \mathrm{~cm}$ wide by $5 \mathrm{~cm}$ high) injection counter are expected to arrive at the end of the magnet. On ejection they come out in a spray horizontally and vertically, such that about $2 \%$ strike a $10 \mathrm{~cm} \times 10 \mathrm{~cm}$ target of the polarization analyzer at 2 meters distance. A vacuum is necessary, of course, in the magnet.

The polarization analyzer is a pulsed field device. It is a null-detector for polarization along the axis of the pulsed magnetic field. A pulse flips the muon spin $\pm 90^{\circ}$ about the axis of the flipping coil, and counter telescopes above and below measure the electron decay rate in a $5 \mu \mathrm{sec}$ gate after the $0.5 \mu \mathrm{sec}$ flipping pulse. The component of spin perpendicular to the coil axis is thus expected to have the form shown in Fig. 4. and the frequency of this variation gives just the $g-2$ precession rate. The data is to be recorded $v s$ muon storage time by means of a Digitronlike device ${ }^{8}$.

\section{PRELIMINARY EXPERIMENT}

Our 6-meter magnet arrived only two months ago, and while we were preparing apparatus to be used with it on its arrival, we took the opportunity to test the techniques involved experimentally on a magnet of $80 \mathrm{~cm}$ length, which was too small to study adiabatic changes of gradient, etc., but good enough for injection, storage, vertical focusing, etc. This was shimmed to give $1.3 \mathrm{~cm} /$ turn and then $2 \mathrm{~mm} /$ turn (about 100 turns) and in fact an experiment to measure $g$-2 was performed as shown in Fig. 5. In this 100-turn magnet-plus-wire experiment we found experimentally $0.15 \%$ of the injected muons to complete 100 turns or more. The distribution of muon intensity at counter $4 v$ s storage time is shown in Fig. 6. All of this loss can be understood from a) running back 
Perpendicular component

of decay asymmetry

standard deviations due

to form of intensity

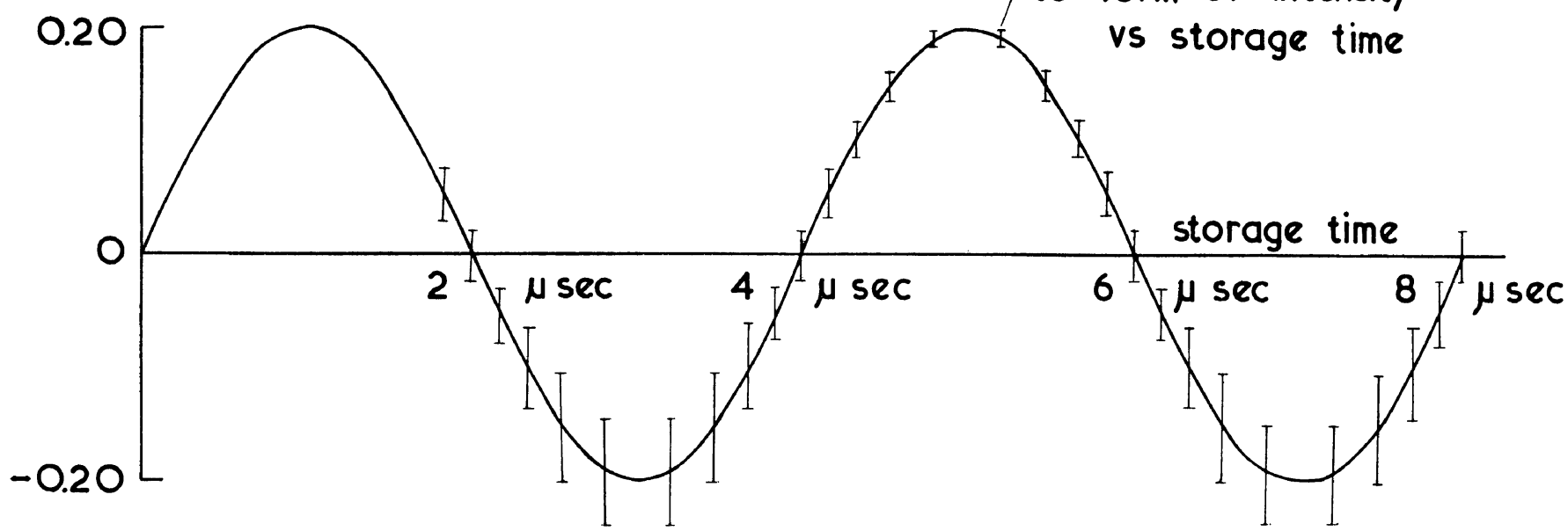

Fig. 4 The time dependence of the magnitude of the component of spin perpendicular to the coil axis.

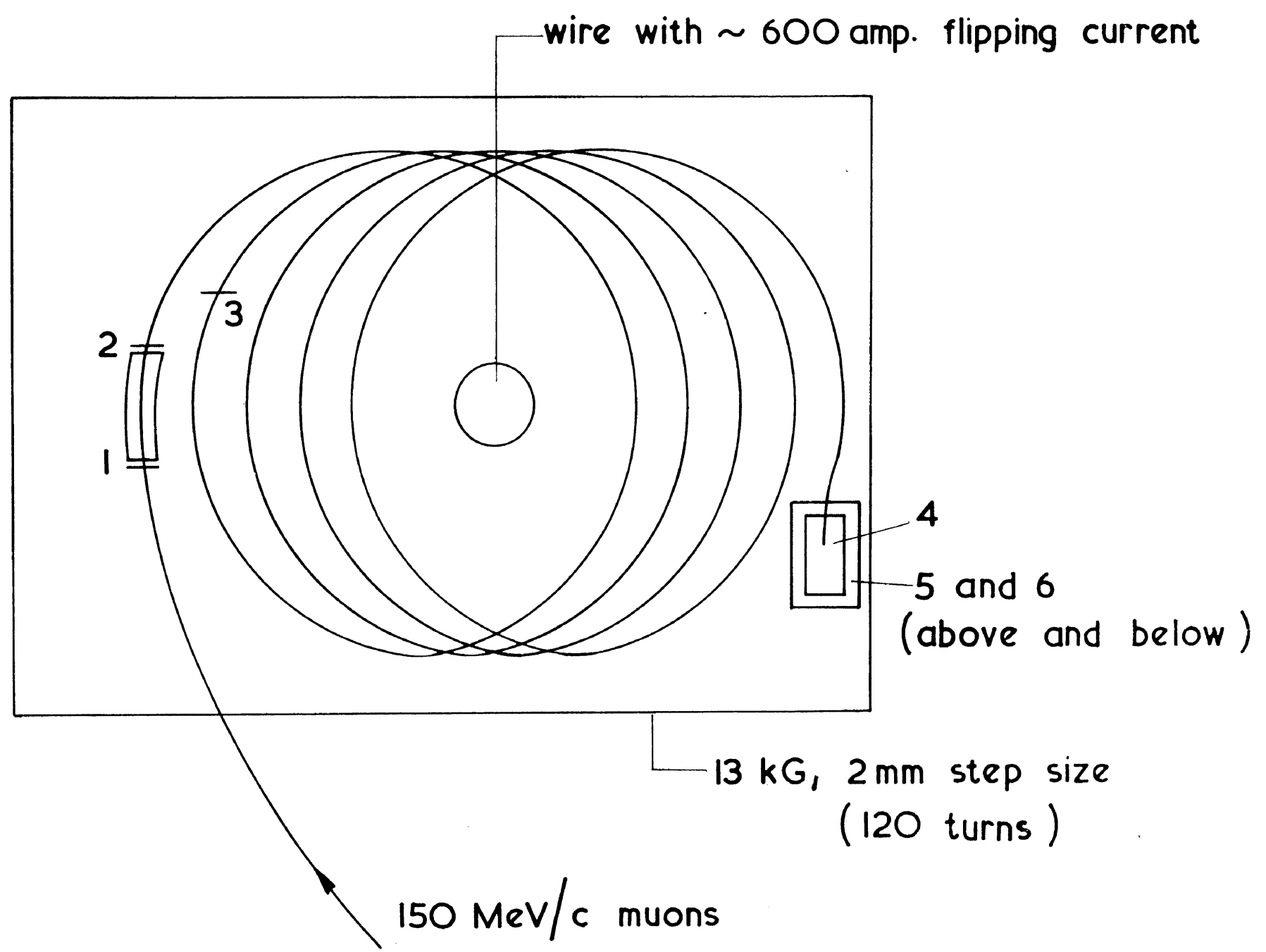

Fig. 5 Diagram of the experiment using the $80 \mathrm{~cm}$ magnet. 

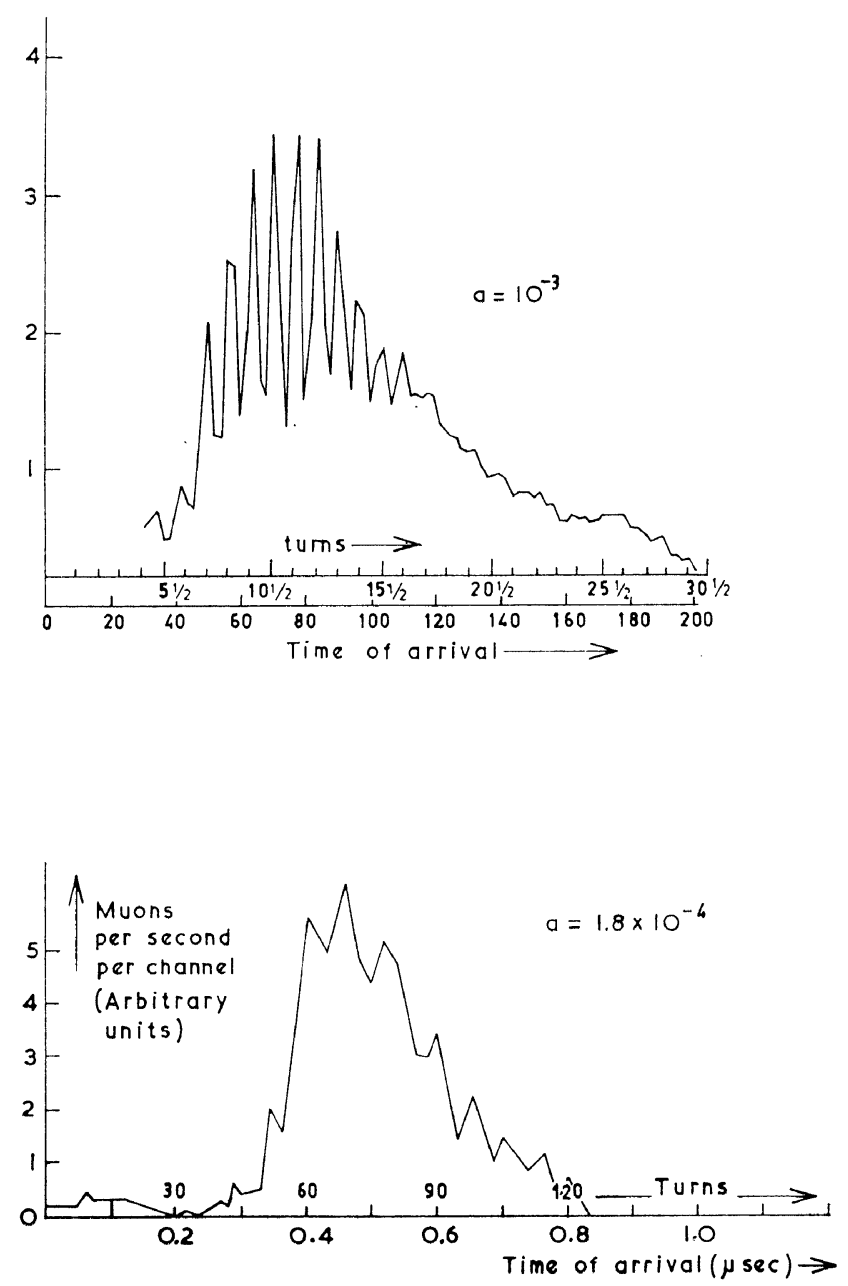

Fig. 6 Distribution of muon intensity at counter $4 v s$. storage time. (a) magnet shimmed to $1.3 \mathrm{~cm} / \mathrm{turn}$; (b) magnet shimmed to $2 \mathrm{~mm} /$ turn.

into the moderator, b) hitting the central wire and returns, c) vertical acceptance loss (factor of 10), d) decay in flight. The accepted angular distribution from the moderator is 0.1 radians wide.

Since it did not seem possible to extract muons from this magnet in any reasonable fashion, we changed the transverse polarization produced by $g-2$ into a vertical component along the field, by means of current in a wire always linked by the orbits. This experiments bears a great similarity to one proposed by M. Schwartz and J. Steinberger in 1957.

We have noted that there exists a current for which an initial transverse polarization is transformed into a transverse polarization so that there is no false $g-2$ effect from this cause, and we have worked at this current. In addition, since the orbits always encircle the wire, the situation is somewhat different from that of Schwartz and Steinberger. Transverse polarization is present in the stored muons even if the beam has no transverse component, since the acceptance is biased toward muons multiply scattered toward the left in the moderator. That is why it is important to work at a current such that one minimizes the sensitivity to the initial transverse component of polarization.

The up-down asymmetry of the decay of muons stopping in counter $4 \mathrm{vs}$. storage time was measured for the two directions of current in the central wire, and subsidiary experiments were done with half of the counter 4 covered, to detect false $g$ - 2 effects produced by effects of the wire current on the orbits. Unfortunately, the polarization measurement is not a null-experiment, and to determine the angle by which the spin has precessed one needs to know the up-down asymmetry which would have been produced by beam muons stopping in the scintillator of counter 4 in 13,000 gauss with their spin along the magnetic field. This was measured in a different magnet, and a static asymmetry obtained by comparison of pions and muons stopping in the same counter 4 . Since each circulating momentum feels a different wire field, the vertical component of the polarization at a given storage time is composed of the vertical component of the polarization of muons of various momenta. A folding process is thus necessary to deduce from assumed ( $g-2)$ values asymmetry data to be compared with the experimental points. To do this, the momentum distribution of stopping and decaying muons must be known. This we determined, as a function of storage time, by pulse height analysis and calibrated by measurements of the orbit radius. Many such investigations were necessary and were performed. The experiment was performed in principle in a satisfactory manner.

The overall result of the experiment gives

$$
(g-2) / 2=\alpha / 2 \pi \times(1.8 \pm 0.3),
$$

but we hasten to add that $\chi^{2}$ of this best-fitting result is 9 instead of 5 , showing that the experiment is afflicted with non-statistical errors of considerable magnitude. The result is completely inconsistent statistically with the QED value of the anomaly. We regard it as a good demonstration of the existence and sign of $g$-2, but we would not claim this number without redoing the experiment to find the cause of the impossibly large $\chi^{2}$. On the other hand, it would have been much harder to make of this a proper 
experiment than to go ahead with the $1 \%$ experiment with the 6-meter magnet, and we regard this as a much better investment of our time.

\section{STATUS}

The techniques of shimming, storage, vacuum, counting, and polarization analysis to be used in the 6-meter experiment have all been experimentally confirmed. At this moment, the shims have all been designed and measured, and one is proceeding to integral measurements of the orbits by means of $\alpha$-particle tests. All these tests have been done before with the smaller magnet, and we hope to have $g$-2 to $1 \%$.

\section{ACKNOWLEDGMENT}

We wish to thank Prof. G. Bernardini for his enthusiastic and continued support of this project from its inception, without which this work would have been impossible. We are greatly indebted to Prof. W. K. H. Panofsky for his many contributions during the fall of 1959 when he visited CERN and Prof. L. M. Lederman for his early work at CERN on this problem.

\title{
LIST OF REFERENCES AND NOTES
}

1. Garwin, R. L., Lederman, L. M., Weinrich, M. Phys. Rev. 105, p. 1415 (1957).

2. Berestetskii, Korkhin and Khlebnikov. JETP 3, p. 761 (1956).

3. Suura, H. and Wichmann, E. H. Phys. Rev. 105, p. 1930 (1957); Petermann, A. Phys. Rev. 105, p. 1931 (1957).

4. Koslov, S., Fitch, V. and Rainwater, J. Phys. Rev. 95, p. 29 (1954).

5. Lathrop, J., Lundy, R. A., Penman, S., Telegdi, V. L., Winston, R., Yovanovitch, D. D. and Bearden, A. J. Nuovo Cimento 17, p. 114 (1960).

6. Garwin, Hutchinson, Penman and Shapiro. Phys. Rev. Letters 2, p. 516 (1959).

7. Nelson, D. F., Schupp, A. A., Pidd, R. W. and Crane, H. R. Phys. Rev. Letters 2, p. 492 (1959).

8. Swanson, R. Rev. Sci. Inst. 31, p. 149 (1960).

\section{MUON MASS AND CHARGE BY CRITICAL ABSORPTION OF MESIC X-RAYS}

\author{
S. Devons, G. Gidal, L. M. Lederman and G. Shapiro \\ Columbia University, New York, New York
}

(presented by L. M. Lederman)

The high precision currently available ${ }^{11}$ in determinations of the muon magnetic moment has stimulated efforts to obtain the mass directly. This may be considered parallel but different from the $(g-2)$ experiment. We report the first results of a study of the $3 D-2 P$ transition in $\mu$-mesic phosphorus, a mesic x-ray known to have energy very close to the $K$-absorption edge of lead ${ }^{2,3,4)}$.
Thus a careful absorption measurement ${ }^{3,4)}$ in $\mathrm{Pb}$ of the radiation from a $\mu^{-}$stopped in phosphorus can yield an absorption coefficient $\mu$, which may be compared to a recent remeasurement of $\mu$ as a function of $\mathrm{x}$-ray energy ${ }^{5}$. If, as we observe, this mesic $\mathrm{x}$-ray line falls on the rapidly rising part of the $\mu$ vs $E$ curve, the energy of the x-ray may be determined to within $0.01 \%$. Since this energy is directly pro- 